\title{
In vitro antibacterial activity of macelignan and corosolic acid against the bacterial bee pathogens Paenibacillus larvae and Melissococcus plutonius
}

\author{
Jaegoo Kim ${ }^{1,5}$, Sangchul Park ${ }^{1,5}$, Yu-Kyong Shin ${ }^{2,5}$, Hee Kang ${ }^{3}$, and Ki-Young Kim ${ }^{1,4}$ \\ Kyung Hee University, ${ }^{1}$ Graduate School of Biotechnology, Kihung, Yongin, Gyeonggi, Republic of Korea, \\ ${ }^{2}$ College of Life Science, Kihung, Yongin, Gyeonggi, Republic of Korea, ${ }^{3} \mathrm{Graduate}$ School of Oriental \\ Medicine, Kihung, Yongin, Gyeonggi, Republic of Korea, ${ }^{4}$ College of Life Science and Graduate School \\ of Biotechnology, Department of Genetic Engineering, Giheung-gu, Yongin-si, Gyeonggi-do, \\ Republic of Korea
}

${ }^{5}$ These authors contributed equally as joint first authors

Received August 4, 2017

Accepted June 27, 2018

\begin{abstract}
Foulbrood disease, which is caused by Paenibacillus larvae (American foulbrood) or Melissococcus plutonius (European foulbrood disease), is a major threat to honeybees (Apis mellifera) worldwide. Tetracycline derivatives have been used to control these bacteria, but resistant strains have evolved, and the antibiotic derivatives can adversely affect bee health. When foulbrood disease is discovered, beekeepers usually burn the bee hives and equipment. The aim of this study was to investigate the in vitro susceptibility of P. larvae and M. plutonius to new antibacterial agents. Antibacterial activities of seven compounds prepared as serial two-fold dilutions were assayed using 96 -well microtitre plates. Minimum inhibitory concentration values were obtained after $24 \mathrm{~h}$ or $48 \mathrm{~h}$ of incubation. Antibacterial synergistic activity of tetracycline and the test compounds was evaluated using broth micro-dilution assays with two-fold serial dilutions of the compounds. Among the seven compounds tested, macelignan and corosolic acid showed the strongest anti-bacterial activity. In addition, tetracycline interacted synergistically with corosolic acid to reduce $P$. larvae and $M$. plutonius growth. Even though macelignan and corosolic acid were worth as solely effective agents to treat $P$. larvae and $M$. plutonius, combinatorial treatment with tetracycline would be more useful to overcome toxicity, resistance occurrence and costliness. Further validation studies of these compounds and identification of their targets, as well as actual field tests and bee toxicity studies are still needed. However, macelignan and corosolic acid as natural secondary metabolites would be effective agents for bee foulbrood disease with valuable antibacterial activities.
\end{abstract}

Honeybee, infectious disease, natural compound, anti-bacterial agent

Approximately one third of the human food supply depends on insect pollination. Honeybees are the most economically important pollinators of agricultural and horticultural plants. Bee populations worldwide have been declining for various reasons (Genersch 2010; Cornman et al. 2012; Seitz et al. 2016). No single factor can account for the overall global decline, but a contributing factor is bacteria, including Paenibacillus larvae and Melissococcus plutonius (Miyagi et al. 2000; Alippi et al. 2002; Takamatsu et al. 2014; Takamatsu et al. 2016).

Paenibacillus larvae is a gram-positive endospore-forming bacterium known to cause American foulbrood (AFB) disease, which is one of the most economically devastating bee diseases. Paenibacillus larvae infects the brood stages of honeybees (Apis mellifera L.), and the infected honeybee brood turns black with a spotted appearance and bitter smell. The brood becomes a hard scale of material that sticks to the walls of the cells. Infected larvae usually die after the cell is sealed, and billions of infective spores form in their remains. Paenibacillus larvae spores remain viable for many years and are highly

Address for correspondence:

Ki-Young Kim, $\mathrm{PhD}$

Department of Genetic Engineering

College of Life Science and Graduate School of Biotechnology

Kyung Hee University

1 Seocheon, Giheung, Yongin, Gyeonggi-do 446-701, Republic of Korea

Phone: +82312012633

E-mail: kiyoung@khu.ac.kr

http://actavet.vfu.cz/ 
infectious and can spread to neighboring hives. They are very resistant to extremes of hot and cold and to many disinfectants. Some regions therefore have a "burn only" policy, but others allow the use of antibiotics to control the disease (Miyagi et al. 2000; Alippi et al. 2002; Genersch 2007; Ebeling et al. 2016).

Melissococcus plutonius is a gram-positive bacterium that causes European foulbrood (EFB) disease. European foulbrood is considered less virulent than AFB, but infected larvae usually die before they are capped (Bailey 1983; Forsgren et al. 2005; Takamatsu et al. 2014). Yearly reoccurrence of EFB from contaminated combs and equipment can occur. Melissococcus plutonius does not produce spores, but combs contaminated with the bacteria can still re-infect honey bees in subsequent years.

Foulbrood is considered a serious bee disease because it can cause massive damage to the apiculture industry (Forsgren 2010; Genersch 2010). The antibiotic oxytetracycline hydrochloride has been used to treat foulbrood (Thomps on and Brown 2001; Thomps on et al. 2005). However, continuous use of this antibiotic has reduced the viability of honeybees (Pettis et al. 2004; Thompson et al. 2005; Jasny 2017; Raymann et al. 2017), increased the chance of presence of antibiotics in honey (Mutinelli 2003), drove the evolution of antibiotic-resistant strains (Alippi et al. 2002; Evans 2003; Jasny 2017) and killed the valuable honey bee bacteria (Yoshiyama et al. 2009; Alberoni et al. 2016; Jasny 2017; Raymann et al. 2017). Those concerns have resulted in a decreased use of this antibiotic by honeybee keepers and have spurred scientists to find safer antibacterial agents that can be used to treat foulbrood.

Natural plants and medicinal herbs have been a valuable source of novel anti-microbial agents, because they have abundant biological activities and are pharmacologically safe (Albo et al. 2003; Özk1rım et al. 2014; González et al. 2015; Shin and Kim 2016). In addition, frequent consumption of plants by humans suggests that many secondary plant metabolites are not toxic to humans (Park et al. 1999; Motsei et al. 2003; Webster et al. 2008; Maggi et al. 2011). We identified macelignan and corosolic acid as putative novel anti-fungal agents against Ascosphaera apis based on the screening of a natural compound library in a previous study (Shin and Kim 2016).

Here, we examined the antibacterial effects of macelignan and corosolic acid, as well as emodin-8-O- $\beta$-D-glucopyranoside, fangchinoline, loganic acid, tracheloside, dehydrocostus lactone, miconazole, congo red and kanamycine against the causative agents of foulbrood to identify new candidate compounds that have effective anti-bacterial activity against $P$. larvae and $M$. plutonius.

\section{Materials and Methods}

\section{Strain maintenance}

Glycerol stock of P. larvae (KCTC14031, Korea) was streaked on MYPGP (MYPGP: 1\% Mueller-Hinton broth, $1.5 \%$ yeast extract, $0.2 \%$ glucose, $0.3 \% \mathrm{~K}_{2} \mathrm{HPO}_{4}$, and $0.1 \%$ sodium pyruvate) agar and cultured at $35^{\circ} \mathrm{C}$ for $48 \mathrm{~h}$. For the broth microdilution assay of $P$. larvae, Mueller Hinton broth with thiamine (MHBT) was used (Gende et al. 2008; CLSI document M100-S21 2009; Maggi et al. 2011). Glycerol stock of M. plutonius (ATCC35311, USA) was streaked on KSBHI medium (BHI medium plus $0.15 \mathrm{M} \mathrm{KH}_{2} \mathrm{PO}_{4}$ and $1 \%$ soluble starch) for 3 days at $35^{\circ} \mathrm{C}$ in a $5 \% \mathrm{CO}$ atmosphere. For broth microdilution assay of $M$. plutonius, KSBHI broth medium was used (Takamatsu et al. 2014; Wu et al. 2014; Takamatsu et al. 2016). Glycerol stocks of other bacteria were streaked on Luria-Bertani (LB) agar $(1 \%(\mathrm{w} / \mathrm{v})$ bacto-tryptone, $0.5 \%(\mathrm{w} / \mathrm{v})$ bacto-yeast extract, $1 \%(\mathrm{w} / \mathrm{v})$ $\mathrm{NaCl}$, and $4.5 \%(\mathrm{w} / \mathrm{v})$ nutrient agar) and incubated aerobically at $37^{\circ} \mathrm{C}$ overnight.

For Staphylococcus aureus (KCTC1621, Korea) and Staphylococcus saprophyticus (KACC15799, Korea), Mueller-Hinton broth (30.0\% beef infusion, $1.75 \%$ casein hyrolysate, and $0.15 \%$ starch adjusted pH7) was used. For Bacillus subtilis subsp. Spizizenii (KCTC3705, Korea), Escherichia coli (KACC11598, Korea), Micrococcus luteus (KACC13377, Korea) and Enterococcus faecalis (KACC11304, Korea), Mueller-Hinton II broth was used.

\section{In vitro antibacterial assay}

Antibacterial activities of each compound were assayed based on the Clinical and Laboratory Standards Institute document (CLSI document M100-S21 2009) with slight modifications using 96-well microtitre plates (Miyagi 
et al. 2000; Ansari et al. 2016; Vukic et al. 2017). Briefly, $10 \mathrm{mg} / \mathrm{ml}$ stock of each compound was prepared in dimethyl sulphoxide (DMSO). Test compounds (Chemface, Wuhan, China) and positive control miconazole (MB cell, Seoul, Korea), Congo red (DCC, Seoul, Korea) and kanamycine (Georgiachem, Georgia, USA), were prepared as serial two-fold dilutions with final concentrations ranging between $0.2-100 \mathrm{mg} / \mathrm{l}$. A single colony of each bacterial isolate was separately inoculated into $3 \mathrm{ml}$ of media. After that, each well was inoculated with each bacterial suspension at a final density of 0.5 McFarland. Minimum inhibitory concentration (MIC) was defined as the lowest concentration of compound that inhibited bacterial growth after $24 \mathrm{~h}$ or $48 \mathrm{~h}$ of incubation. Wells without any compounds were used as negative controls of growth. This experiment was repeated at least three times, and duplicate samples were prepared.

\section{Antibacterial synergy testing}

To assess if the test compounds and tetracycline had synergistic antibacterial activity, a broth microdilution assay was performed using a checkerboard design (Drogari-Apiranthitou et al. 2012; Shin and Kim 2016). Tetracycline was serially two-fold diluted, and test compounds were also vertically serially two-fold diluted and dispensed into a 96-well microtitre plate. One hundred microlitres of P. larvae or M. plutonius suspension at a density of $0.5 \mathrm{McF}$ arland were placed in the wells. Final drug concentrations ranged from $10 \mathrm{mg} / 1$ to $0.01 \mathrm{mg} / 1$ tetracycline, $25 \mathrm{mg} / 1$ to $0.02 \mathrm{mg} / \mathrm{l}$ corosolic acid, and $12.5 \mathrm{mg} / 1$ to $0.1 \mathrm{mg} / \mathrm{l}$ macelignan. The MIC values of test compounds were individually determined on the same plate as the controls. Fractional inhibitory concentration indexes (FICIs) were used to assess if different combinations of compounds had synergistic effects. Tests were performed at least three times.

\section{Results}

Macelignan and corosolic acid showed antibacterial activity against P. larvae and M. plutonius

We tested the anti-P. larvae and anti-M. plutonius activity of seven compounds (loganic acid, macelignan, tracheloside, fangchinoline, corosolic acid, dehydrocostus lactone, and emodin-8-O- $\beta$-D-glucopyranoside) (Shin and Kim 2016). Of these seven compounds, macelignan had the strongest anti- $P$. larvae activity (MIC of $1.56 \mathrm{mg} / \mathrm{l}$ after a $24 \mathrm{~h}$ incubation and MIC of $3.125 \mathrm{mg} / \mathrm{l}$ after a $48 \mathrm{~h}$ incubation), and corosolic acid had comparable anti- $P$. larvae activity (MIC of $3.125 \mathrm{mg} / \mathrm{l}$ after $24 \mathrm{~h}$ and $48 \mathrm{~h}$ of incubation). Macelignan and corosolic acid also had high anti-M. plutonius activity (MIC of $3.125 \mathrm{mg} / \mathrm{l}$ at $24 \mathrm{~h}$ and $48 \mathrm{~h}$ incubation). The synthetic antifungal agents miconazole $(1.56 \sim 3.125 \mathrm{mg} / \mathrm{l})$ and tetracycline $(0.39 \sim 1.56 \mathrm{mg} / \mathrm{l})$ showed very high antibacterial activity against both strains, but loganic acid, tracheloside, fangchinoline, dehydrocostus lactone, emodin-8-O- $\beta$-Dglucopyranoside, kanamycin, and congo red showed very little or no anti-bacterial activity (Table 1).

Table 1 . Susceptibility of $P$. larvae and M. plutonius to the tested compounds.

\begin{tabular}{lcccc}
\hline Compound & \multicolumn{2}{c}{ P. larvae } & \multicolumn{2}{c}{ M. plutonius } \\
\cline { 2 - 5 } & $24 \mathrm{~h}$ & $48 \mathrm{~h}$ & $24 \mathrm{~h}$ & $48 \mathrm{~h}$ \\
\hline Loganic acid & $>200$ & $>200$ & $>200$ & $>200$ \\
Macelignan & 1.56 & 3.125 & 3.125 & 3.125 \\
Tracheloside & $>200$ & $>200$ & $>200$ & $>200$ \\
Fangchinoline & 50 & $>200$ & $>200$ & $>200$ \\
Corosolic acid & 3.125 & 3.125 & 3.125 & 3.125 \\
Dehydrocostus lactone & 50 & 50 & $>200$ & $>200$ \\
Emodin-8-O-B-D-glucopyranoside & $>200$ & $>200$ & $>200$ & $>200$ \\
Miconazole & 1.56 & 3.125 & 3.125 & 3.125 \\
Congo red & $>200$ & $>200$ & $>200$ & $>200$ \\
Kanamycin & $>200$ & $>200$ & $>200$ & $>200$ \\
Tetracycline & 0.78 & 1.56 & 0.39 & 0.78 \\
\hline
\end{tabular}

Minimum inhibitory concentration (MIC) values in $\mathrm{mg} / \mathrm{l}$ 
Next, we investigated if macelignan had antibacterial activity against a broad spectrum of human pathogens. Macelignan had anti-bacterial activity against B. subtilis subsp. spizizenii, M. luteus, M. plutonius, and P. larvae (MICs of $25 \mathrm{mg} / 1,12.5 \mathrm{mg} / 1,3.125$ $\mathrm{mg} / \mathrm{l}$, and $3.125 \mathrm{mg} / \mathrm{l}$ after $48 \mathrm{~h}$ incubation, respectively) (Table 2). We also investigated if corosolic acid had broad spectrum antibacterial activity. Corosolic acid showed antibacterial activity against $M$. luteus, $M$. plutonius, and $S$. saprophyticus $(6.25 \mathrm{mg} / 1,3.125$ $\mathrm{mg} / \mathrm{l}$, and $50 \mathrm{mg} / \mathrm{l}$ at $48 \mathrm{~h}$, respectively; Table 3 ). In addition, tetracycline showed very high anti-bacterial activity against $B$. subtilis subsp. spizizenii, E. coli, M. luteus, M. plutonius, P. larvae, and S. saprophyticus (MIC values of $0.78 \mathrm{mg} / 1,3.125 \mathrm{mg} / 1,0.39 \mathrm{mg} / 1,0.78 \mathrm{mg} / \mathrm{l}$, $3.125 \mathrm{mg} / \mathrm{l}$, and $3.125 \mathrm{mg} / \mathrm{l}$ after $48 \mathrm{~h}$, respectively) (Table 4).

Corosolic acid and tetracycline showed synergistic anti-bacterial activity

We next investigated if tetracycline and macelignan or corosolic acid had synergistic suppressive effects against $P$. larvae and $M$. plutonius by performing broth microdilution assays using a checkerboard design (Tables 5, 6, 7, 8). Tetracycline did not interact synergistically with macelignan to reduce $P$. larvae and $M$. plutonius growth $(\mathrm{FICI}=0.83$ \pm 0.14 and $0.67 \pm 0.29$, respectively). In contrast, tetracycline interacted synergistically with corosolic acid to reduce $P$. larvae and M. plutonius growth (FICI $=0.29 \pm 0.07$ and $0.41 \pm 0.14$, respectively).

Table 2. Susceptibility of various bacteria to macelignan.

\begin{tabular}{llcr}
\hline & Strain name & \multicolumn{2}{c}{ MIC } \\
\cline { 2 - 3 } KCTC3705 & B. subtilis subsp. spizizenii & $24 \mathrm{~h}$ & $48 \mathrm{~h}$ \\
KACC11598 & E. coli & 1.56 & 25 \\
KACC11304 & E. faecalis & $>200$ & $>200$ \\
KACC13377 & M. luteus & $>200$ & $>200$ \\
ATCC35311 & M. plutonius & 12.5 & 12.5 \\
KACC14031 & P. larvae & 3.125 & 3.125 \\
KCTC1621 & S. aureus & 1.56 & 3.125 \\
KACC15799 & S. saprophyticus & $>200$ & $>200$ \\
\hline
\end{tabular}

Minimum inhibitory concentration (MIC) values in $\mathrm{mg} / \mathrm{l}$

Table 3. Susceptibility of various bacteria to corosolic acid.

\begin{tabular}{llcr}
\hline & Strain name & \multicolumn{2}{c}{ MIC } \\
\cline { 3 - 4 } KCTC3705 & B. subtilis subsp. spizizenii & $24 \mathrm{~h}$ & $48 \mathrm{~h}$ \\
KACC11598 & E. coli & 6.25 & 200 \\
KACC11304 & E. faecalis & $>200$ & $>200$ \\
KACC13377 & M. luteus & $>200$ & $>200$ \\
ATCC35311 & M. plutonius & 6.25 & 6.25 \\
KACC14031 & P. larvae & 3.125 & 3.125 \\
KCTC1621 & S. aureus & 3.125 & $>200$ \\
KACC15799 & S. saprophyticus & 7200 & 50 \\
\hline
\end{tabular}

Minimum inhibitory concentration (MIC) values in $\mathrm{mg} / 1$ 
Table 4. Susceptibility of various bacteria to tetracycline.

\begin{tabular}{llcc}
\hline & Strain name & \multicolumn{2}{c}{ MIC } \\
\cline { 3 - 4 } KCTC3705 & B. subtilis subsp. spizizenii & $24 \mathrm{~h}$ & $48 \mathrm{~h}$ \\
KACC11598 & E. coli & 0.39 & 0.78 \\
KACC11304 & E. faecalis & 3.125 & 3.125 \\
KACC13377 & M. luteus & 100 & 200 \\
ATCC35311 & M. plutonius & 0.39 & 0.39 \\
KACC14031 & P. larvae & 0.39 & 0.78 \\
KCTC1621 & S. aureus & 1.56 & 3.125 \\
KACC15799 & S. saprophyticus & 200 & 200 \\
\hline
\end{tabular}

Minimum inhibitory concentration (MIC) values in mg/l

Table 5. Synergistic effects of macelignan and tetracycline against $P$. larvae.

\begin{tabular}{lrrrrrc}
\hline P. larvae (P618) & \multicolumn{4}{c}{ MIC $^{1}$} & \multicolumn{2}{c}{ FICI $^{2}$} \\
\cline { 2 - 7 } & \multicolumn{2}{c}{ Macelignan } & \multicolumn{2}{c}{ Tetracycline } & \multicolumn{2}{c}{ Macelignan + Tetracycline } \\
\cline { 2 - 7 } & $24 \mathrm{~h}$ & $48 \mathrm{~h}$ & $24 \mathrm{~h}$ & $48 \mathrm{~h}$ & $24 \mathrm{~h}$ & $48 \mathrm{~h}$ \\
\hline Median & 1.56 & 3.125 & 0.78 & 1.56 & $0.83 \pm 0.14$ & $0.83 \pm 0.14$ \\
\hline
\end{tabular}

${ }^{1}$ Minimum inhibitory concentration (MIC) values in $\mathrm{mg} / \mathrm{l}$

${ }^{2}$ Fractional inhibitory concentration index (FICI)

Table 6. Synergistic effects of corosolic acid and tetracycline against $P$. larvae.

\begin{tabular}{llccccc}
\hline P. larvae (P618) & \multicolumn{4}{c}{ MIC $^{1}$} & \multicolumn{2}{c}{ FICI $^{2}$} \\
\cline { 2 - 7 } & \multicolumn{2}{c}{ Corosolic acid } & \multicolumn{2}{c}{ Tetracycline } & \multicolumn{2}{c}{ Corosolic acid + Tetracycline } \\
\cline { 2 - 7 } & $24 \mathrm{~h}$ & $48 \mathrm{~h}$ & $24 \mathrm{~h}$ & $48 \mathrm{~h}$ & $24 \mathrm{~h}$ & $48 \mathrm{~h}$ \\
\hline Median & 3.13 & 3.13 & 0.78 & 0.78 & $0.29 \pm 0.07$ & $0.46 \pm 0.07$ \\
\hline
\end{tabular}

${ }^{1}$ Minimum inhibitory concentration (MIC) values in $\mathrm{mg} / \mathrm{l}$

${ }^{2}$ Fractional inhibitory concentration index (FICI)

Table 7. Synergistic effects of macelignan and tetracycline against M. plutonius.

\begin{tabular}{lrrrrrr}
\hline M. plutonius & \multicolumn{4}{c}{ MIC $^{1}$} & \multicolumn{2}{c}{ FICI $^{2}$} \\
\cline { 2 - 7 } & \multicolumn{2}{c}{ Macelignan } & \multicolumn{2}{c}{ Tetracycline } & Macelignan + Tetracycline \\
\cline { 2 - 7 } & $24 \mathrm{~h}$ & $48 \mathrm{~h}$ & $24 \mathrm{~h}$ & $48 \mathrm{~h}$ & $24 \mathrm{~h}$ & $48 \mathrm{~h}$ \\
\hline Median & 3.125 & 3.125 & 0.39 & 0.78 & $0.67 \pm 0.29$ & $1.0 \pm 0$ \\
\hline
\end{tabular}

${ }^{1}$ Minimum inhibitory concentration (MIC) values in $\mathrm{mg} / \mathrm{l}$

${ }^{2}$ Fractional inhibitory concentration index (FICI)

\section{Discussion}

Tetracyclines are widely used as broad-spectrum antibiotics, but their use has decreased because of resistance development (Chopra and Roberts 2001). Oxytetracycline is a tetracycline derivative that has been used to treat infectious bacterial bee diseases such as 
Table 8. Synergistic effects of corosolic acid and tetracycline against $M$. plutonius.

\begin{tabular}{lrrrrrr}
\hline M. plutonius & \multicolumn{4}{c}{ MIC $^{1}$} & \multicolumn{2}{c}{ FICI $^{2}$} \\
\cline { 2 - 7 } & \multicolumn{2}{c}{ Corosolic acid } & \multicolumn{2}{c}{ Tetracycline } & \multicolumn{2}{c}{ Corosolic acid + Tetracycline } \\
\cline { 2 - 7 } & $24 \mathrm{~h}$ & $48 \mathrm{~h}$ & $24 \mathrm{~h}$ & $48 \mathrm{~h}$ & $24 \mathrm{~h}$ & $48 \mathrm{~h}$ \\
\hline Median & 3.125 & 3.125 & 0.39 & 0.78 & $0.41 \pm 0.14$ & $0.33 \pm 0.14$ \\
\hline
\end{tabular}

${ }^{1}$ Minimum inhibitory concentration (MIC) values in $\mathrm{mg} / \mathrm{l}$

${ }^{2}$ Fractional inhibitory concentration index (FICI)

AFB and EFB, but resistance has been reported (Alippi 1996; Miyagi et al. 2000; Evans 2003).

Commercially available antimicrobial compounds usually inhibit the growth of various microorganisms, including beneficial bacteria. If broad range antimicrobial agents such as tetracycline are used to treat pathogens, the growth of favourable organisms will also be affected. Moreover, there is a possibility of the presence of tetracyline in honey (Mutinelli 2003), which could cause the inhibition of favourable organisms in the consumer. Therefore, there is currently a focus on identifying narrow-range antimicrobial agents (Bax and Green 2015).

In this study, macelignan and corosolic acid showed the strongest anti-bacterial activity among the seven compounds we tested. In addition, macelignan and corosolic acid had narrow antimicrobial activity ranges. We previously reported that macelignan had antifungal activity by inhibiting fungal mitogen-activated protein kinase phosphorylation (Shin and Kim 2016). Moreover, macelignan showed antibacterial and sporicidal activity against B. cereus (Rukayadi et al. 2009). When macelignan was combined with 1,2-hexanediol, an alternative preservative in cosmetics without any side effect, a highly synergistic effect was observed as food-grade antimicrobials (Yogiara et al. 2015).

Corosolic acid is usually extracted from the banaba (Lagerstroemia speciosa) leaf. It is reported to exhibit antihyperlipidemic, antioxidant, antiinflammatory, antiviral, antineoplastic, osteoblastic, and protein kinase C inhibition activity (Kim et al. 2016). Several studies have reported its antimicrobial activity using plant extract or isolated corosolic acid, which showed similar activity compared with our results. In addition, no adverse effects have been observed or reported in animal studies or controlled human clinical trials (Stohs et al. 2013).

In our previous study (Shin and Kim 2016), macelignan and corosolic acid also showed antifungal activity, which suggests that macelignan and corosolic acid can be used to treat A. apis infections. Together, these results suggest that macelignan and corosolic acid show a potential for the treatment of bee-related infectious fungal and bacterial diseases.

Combinatorial treatment of $P$. larvae and M. plutonius with tetracycline and corosolic acid resulted in enhanced anti-bacterial activity compared to that of either compound alone. Combined treatment of bees with tetracycline and corosolic acid might therefore reduce the development of resistance and reduce costs. However, there is still a weak point because macelignan and corosolic acid could kill only vegetative stages, not spores. Thus, these substances should be combined with appropriate beehive disinfection such as heat treatments, use of disinfectants (Dobbelaere et al. 2001) or transfer of bees to clean beehives.

In conclusion, macelignan and corosolic acid showed a strong antibacterial activity against $P$. larvae and $M$. plutonius and a narrow spectrum of antibacterial activity against other bacteria. Corosolic acid and tetracycline had suppressive effects on bacterial growth. In a future study, we plan to determine the appropriate ratio of these two compounds to 
maximize their anti-fungal and anti-bacterial activities and then perform a field test to confirm the effectiveness of these compounds.

As natural compounds, macelignan and corosolic acid should have minimal impact on the environment and are promising candidates for treating microbial bee diseases.

\section{Conflict of Interest}

This research was conducted in the absence of any commercial or financial relationships that could be construed as posing potential conflicts of interest.

\section{Acknowledgements}

This research was supported by the Basic Science Research Program through the National Research Foundation of Korea (NRF) funded by the Ministry of Education (NRF-2015R1D1A1A01061225) and the IPET (116094-03-1-SB010).

\section{References}

Alberoni D, Gaggìa F, Baffoni L, Di Gioia D 2016: Beneficial microorganisms for honey bees: problems and progresses. Appl Microbiol Biotechnol 100: 9469-9482

Albo GN, Henning C, Ringuelet J, Reynaldi FJ, De Giusti MR, Alippi AM 2003: Evaluation of some essential oils for the control and prevention of American Foulbrood disease in honey bees. Apidologie 34: 417-427

Alippi AM 1996: Characterization of isolates of Paenibacillus larvae with biochemical type and oxytetracycline resistance. Rev Argent Microbiol 28: 197-203

Alippi AM, López AC, Aguilar OM 2002: Differentiation of Paenibacillus larvae subsp. larvae, the cause of American foulbrood of honeybees, by using PCR and restriction fragment analysis of genes encoding $16 \mathrm{~S}$ rRNA. Appl Environ Microbiol 68: 3655-3660

Ansari MJ, Al-Ghamdi A, Usmani S, Al-Waili N, Nuru A, Sharma D, Khan KA, Kaur M, Omer M 2016: In vitro evaluation of the effects of some plant essential oils on Paenibacillus larvae, the causative agent of American foulbrood. Biotechnol Biotechnol Equip 30: 49-55

Bailey L 1983: Melissococcus pluton, the cause of European foulbrood of honey bees (Apis spp.). J Appl Microbiol 55: 65-69

Bax R, Green S 2015: Antibiotics: the changing regulatory and pharmaceutical industry paradigm. J Antimicrob Chemother 70: 1281-1284

Chopra I, Roberts M 2001: Tetracycline antibiotics: mode of action, applications, molecular biology, and epidemiology of bacterial resistance. Microbiol Mol Biol Rev 65: 232-260

Clinical and Laboratory Standard Institute, Performance standard for antimicrobial susceptibility testing; $21 \mathrm{st}$ informational supplement, CLSI document M100-S21 2009. CLSI, Wayne, PA

Cornman RS, Tarpy DR, Chen Y, Jeffreys L, Lopez D, Pettis JS, van Engelsdorp D, Evans JD 2012: Pathogen webs in collapsing honey bee colonies. Plos One 7: e43562

Dobbelaere W, de Graaf DC, Reybroeck W, Desmedt E, Peeters JE, Jacobs FJ. 2001: Disinfection of wooden structures contaminated with Paenibacillus larvae subsp. larvae spores. J Appl Microbiol 91:212-216

Drogari-Apiranthitou M, Mantopoulou FD, Skiada A, Kanioura L, Grammatikou M, Vrioni G Mitroussia-Ziouva A, Tsakris A, Petrikkos G 2012: In vitro antifungal susceptibility of filamentous fungi causing rare infections: synergy testing of amphotericin B, posaconazole and anidulafungin in pairs. J Antimicrob Chemother 67: 1937-1940

Ebeling J, Knispel H, Hertlein G, Fünfhaus A, Genersch E 2016: Biology of Paenibacillus larvae, a deadly pathogen of honey bee larvae. Appl Microbiol Biotechnol 100: 7387-7395

Evans JD 2003: Diverse origins of tetracycline resistance in the honey bee bacterial pathogen Paenibacillus larvae. J Invertebr Pathol 83: 46-50

Forsgren E 2010: European foulbrood in honey bees. J Invertebr Pathol 103: S5-S9

Forsgren E, Lundhagen AC, Imdorf A, Fries I 2005: Distribution of Melissococcus plutonius in honey bee colonies with and without symptoms of European foulbrood. Microb Ecol 50: 369-374

Gende LB, Eguaras MJ, Fritz R 2008: Evaluation of culture media for Paenibacillus larvae applied to studies of antimicrobial activity. Rev Argent Microbiol 40: 147-150

Genersch E 2007: Paenibacillus larvae and American foulbrood in honeybees. Berl Munch Tierarztl Wochenschr 120: 26-33

Genersch E 2010: Honey bee pathology: current threats to honey bees and beekeeping. Appl Microbiol Biotechnol 87: 87-97

González MJ, Beoletto VG, Agnese AM, Audisio MC, Marioli JM 2015: Purification of substances from Achyrocline satureioides with inhibitory activity against Paenibacillus larvae, the causal agent of American foulbrood in honeybees' larvae. Appl Biochem Biotechnol 175: 3349-3359 
Jasny BR 2017: Common antibiotic hurts bee survival. Science 355: 1387

Kim SJ, Cha JY, Kang HS, Lee JH, Lee JY, Park JH, Bae JH, Song DK, Im SS 2016: Corosolic acid ameliorates acute inflammation through inhibition of IRAK-1 phosphorylation in macrophages. BMB Rep 49: 276-281

Maggi M, Gende L, Russo K, Fritz R, Eguaras M 2011: Bioactivity of Rosmarinus officinalis essential oils against Apis mellifera, Varroa destructor and Paenibacillus larvae related to the drying treatment of the plant material. Nat Prod Res 25: 397-406

Miyagi T, Peng CY, Chuang RY, Mussen EC, Spivak MS, Doi RH 2000: Verification of oxytetracycline-resistant American foulbrood pathogen Paenibacillus larvae in the United States. J Invertebr Pathol 75: 95-96

Motsei ML, Lindsey KL, van Staden J, Jäger AK 2003: Screening of traditionally used South African plants for antifungal activity against Candida albicans. J Ethnopharmacol 86: 235-241

Mutinelli F 2003: European legislation governing the authorisation of veterinary medical products with particular reference to the use of drugs for the control of honey bee diseases. Apiacta 38: 156-168

Özkırım A, Keskin N, Kürkçüoğlu M, Başer KHC 2014: Evaluation of some essential oils as alternative antibiotics against American foulbrood agent Paenibacillus larvae on honey bees Apis mellifera L. J Essent Oil Res 24: 465-470

Park KS, Kang KC, Kim JH, Adams DJ, Johng TN, Paik YK 1999: Differential inhibitory effects of protoberberines on sterol and chitin biosyntheses in Candida albicans. J Antimicrob Chemother 43: 667-674

Pettis JS, Kochansky J, Feldlaufer MF 2004: Larval Apis mellifera L: (Hymenoptera: Apidae) mortality after topical application of antibiotics and dusts. J Econ Entomol 97: 171-176

Raymann K, Shaffer Z, Moran NA 2017: Antibiotic exposure perturbs the gut microbiota and elevates mortality in honeybees. Plos Biol 15: e2001861

Rukayadi Y, Lee K, Han S, Kim S, Hwang JK 2009: Antibacterial and sporicidal activity of macelignan isolated from nutmeg (Myristica fragrans Houtt.) against Bacillus cereus. Food Sci Biotechnol 18: 1301-1304

Seitz N, Traynor KS, Steinhauer N, Rennich K, Wilson ME, Ellis JD, Rose R, Tarpy DR, Sagili RR, Caron DM, Delaplane KS, Rangel J, Lee K, Baylis K, Wilkes JT, Skinner JA, Pettis JS, van Engelsdorp D 2016: A national survey of managed honey bee 2014-2015 annual colony losses in the USA. J Apic Res 54: 1-12

Shin YK, Kim KY 2016: Macelignan inhibits bee pathogenic fungi Ascophaera apis growth through HOG1 pathway. Braz J Med Biol Res 49: e5313

Stohs SJ, Miller H, Kaats GR 2013: A review of the efficacy and safety of banaba (Lagerstroemia speciosa L.) and corosolic acid. Phytother Res 26: 317-324

Takamatsu D, Morinishi K,Arai R, SakamotoA, Okura M, Osaki M2014: Typing of Melissococcusplutonius isolated from European and Japanese honeybees suggests spread of sequence types across borders and between different Apis species. Vet Microbiol 171: 221-226

Takamatsu D, Sato M, Yoshiyama M 2016: Infection of Melissococcus plutonius clonal complex 12 strain in European honeybee larvae is essentially confined to the digestive tract. J Vet Med Sci 78: 29-34

Thompson HM, Brown MA 2001: Is contact colony treatment with antibiotics an effective control for European foulbrood? Bee World 82: 130-138

Thompson HM, Waite RJ, Wilkins S, Brown MA, Bigwood T, Shaw M, Ridgway C, Sharman M 2005: Effects of European foulbrood treatment regime on oxytetracycline levels in honey extracted from treated honey bee (Apis mellifera) colonies and toxicity to brood. Food Addit Contam 22: 573-578

Vukic MD, Vukovic NL, Djelic GT, Popovic SL, Zaric MM, Baskic DD, Krstic GB, Tesevic VV, Kacaniova MM 2017: Antibacterial and cytotoxic activities of naphthoquinone pigments from Onosma visianii Clem. EXCLI J 16: $73-88$

Wu M, Sugimura Y, Iwata K, Takaya N, Takamatsu D, Kobayashi M, Taylor D, Kimura K, Yoshiyama M 2014 : Inhibitory effect of gut bacteria from the Japanese honey bee, Apis cerana japonica, against Melissococcus plutonius, the causal agent of European foulbrood disease. J Insect Sci 14: 129

Webster D, Taschereau P, Belland RJ, Sand C, Rennie RP 2008: Antifungal activity of medicinal plant extracts; preliminary screening studies. J Ethnopharmacol 115: 140-146

Yogiara, Hwang SJ, Park S, Hwang JK, Pan JG 2015: Food-grade antimicrobials potentiate the antibacterial activity of 1,2-hexanediol. Lett Appl Microbiol. 60:431-439

Yoshiyama M, Kimura K 2009: Bacteria in the gut of Japanese honeybee, Apis cerana japonica, and their antagonistic effect against Paenibacillus larvae, the causal agent of American foulbrood. J Invertebr Pathol 102: $91-96$ 